KEK-TH-738

hep-th/0101100

JANUARY, 2001

\title{
A Dynamical Solution of Stable Starobinsky-Type Inflationary Model in Quantum Geometry
}

\author{
KEN-Ji HAMADA円 \\ Institute of Particle and Nuclear Studies, \\ High Energy Accelerator Research Organization (KEK), \\ Tsukuba, Ibaraki 305-0801, Japan
}

\begin{abstract}
Quantum geometry gives a regularization scheme-independent effective action, whoes equation of motion for the conformal mode has a stable de Sitter solution at the high-energy region where the coupling of the self-interactions of the traceless mode can be neglected because of the asymptotic freedom. However, the dynamics of the traceless mode suggests that inflation ends at the low-energy region.
\end{abstract}

\footnotetext{
${ }^{1}$ E-mail address : hamada@post.kek.jp
} 
Inflationary scenarios have been studied as the most simple idea to solve both the flatness and the horizon problems naturally [1]-[7]. However, standard inflationary models [1, 2] have demerits, such as we must introduce an unsatisfactory quantity like inflaton and need a fine-tuning to get a quantitatively reasonable scenarios. On the other hand, there is an alternative model by Starobinsky [3], who propose a scenario that inflation is driven dynamically due to one-loop corrections by quantized matter fields. He originally consider unstable de Sitter phase by adjusting the regularization shemedependent term properly because inflation has to end at the low-energy phase. However, even in this scenario, a fine-tuning of the scheme-dependent parameter is necessary to obtain sufficiently long lifetime [4, 5].

The inflation models mentioned above are based on semi-classical arguments. On the other hand, it is believed that quantum geometry solve many problems in quantum cosmology [8, 9]. In this letter we show that quantum geometry can really solve such fine-tuning problems. The idea is based on the recent developments [10, 11] in which we show that the effective action of 4D quantum geometry has a regularization scheme-independent form and may be uniquely determined according to conformal matter contents. The equation of motion of this effective action has a stable de Sitter solution. A mechanism to end the inflationary phase is given by the dynamics of the traceless.

Let us first summarize the results given in ref. [10]. The form of effective action of 4D quantum geometry is strongly constrained by diffeomorphism invariance/background-metric independence [11, 10]. It is because a general coordinate transformation in quantum geometry is related to a conformal change of the background metric so that the integrability condition [12, 13] of conformal anomalies [14, 15, 16] plays an important role to determine the effective action. Really, as discussed in [11, 10, 17, 18], the integrability condition of conformal anomalies not only restricts matter fields to be conformally invariant, but also determines many indefiniteness in the gravity sector. To preserve diffeomorphism invariance at the quantum level, we must add the Wess-Zumino action to an invariant action and quantize such a combined action as a tree action in a self-consistent mannar [19, 20, 21, 11, 10]. The scheme-dependent terms which appear in loop effects of the combined theory cancel with the corresponding terms in the Wess-Zumino action, and then diffeomorphism invariance is realized [10].

We consider a perturbation theory in which the traceless mode is ex- 
panded by the dimensionless coupling, $t$, as $\bar{g}_{\mu \nu}=\left(\hat{g} e^{t h}\right)_{\mu \nu}$ [22, 21, 11, 10]. Here, the metric is decomposed as $g_{\mu \nu}=e^{2 \phi} \bar{g}_{\mu \nu}$. On the other hand, the conformal mode, $\phi$, is evaluated exactly, because we treat conformal anomalies. Thus we obtain a regularization scheme-independent form of $4 \mathrm{D}$ quantum geometry effective action (in Lorentzian signature) [10, 2]

$$
\Gamma=\frac{1}{(4 \pi)^{2}} \int d^{4} x \sqrt{-g}\left\{\frac{f}{4} C_{\mu \nu \lambda \sigma} \log \left(\frac{\Delta_{4}^{C}}{\mu^{4}}\right) C^{\mu \nu \lambda \sigma}-\frac{e}{8} \mathcal{G} \frac{1}{\Delta_{4}} \mathcal{G}\right\}+I_{L E},
$$

where $I_{L E}$ represents lower derivative actions which include actions of conformally invariant matter fields and the Einstein-Hilbert action. $C_{\mu \nu \lambda \sigma}$ is the Weyl tensor and $\Delta_{4}^{C}=\square^{2}+\cdots$ is an appropriate conformally covariant operator for the Weyl tensor. The explicit form of $\Delta_{4}^{C}$ is unknown, but it is known that the conformal variation of this term produces the square of the Weyl tensor [15, 23, 24]. $\mathcal{G}$ is defined by the following combination:

$$
\mathcal{G}=G-\frac{2}{3} \square R
$$

where $G$ is the Euler density defined by

$$
G=R_{\mu \nu \lambda \sigma} R^{\mu \nu \lambda \sigma}-4 R_{\mu \nu} R^{\mu \nu}+R^{2} .
$$

$\Delta_{4}$ is the conformally covariant 4 th order operator [17]

$$
\Delta_{4}=\square^{2}+2 R^{\mu \nu} \nabla_{\mu} \nabla_{\nu}-\frac{2}{3} R \square+\frac{1}{3}\left(\nabla^{\mu} R\right) \nabla_{\mu},
$$

which satisfies $\Delta_{4}=e^{-4 \phi} \bar{\Delta}_{4}$ locally for a scalar.

The coefficients $f$ and $e$ are scheme-independent. They are expanded by the renormalized coupling, $t_{r}$, as

$$
f=f_{0}+f_{1} t_{r}^{2}+\cdots, \quad e=e_{0}+e_{1} t_{r}^{2}+\cdots .
$$

Here, $f_{0}$ and $e_{0}$ have already been computed by one-loop diagrams as

$$
\begin{aligned}
& f_{0}=-\frac{N_{X}}{120}-\frac{N_{W}}{40}-\frac{N_{A}}{10}-\frac{199}{30}+\frac{1}{15}, \\
& e_{0}=\frac{N_{X}}{360}+\frac{11 N_{W}}{720}+\frac{31 N_{A}}{180}+\frac{87}{20}-\frac{7}{90},
\end{aligned}
$$

\footnotetext{
${ }^{2}$ The scheme-dependent term has a non-invariant form, $\bar{R}^{2}$, which have to cancel out from the requirement of diffeomorphism invariance. While an invariant $R^{2}$ term might appear at least from order $t_{r}^{4}$ [11.
} 
where the first three contributions of each coefficient come from $N_{X}$ conformal scalar fields, $N_{W}$ Weyl fermions and $N_{A}$ gauge fields, respectively [16]. The fourth and the last ones come from the traceless mode [25] and the conformal mode [20], respectively. The coefficients $f_{1}$ and $e_{1}$ are contributions from not only two-loop diagrams, but also one-loop diagrams of order $t_{r}^{2}$ which include vertices of the Wess-Zumino action [11].

The beta function for the coupling, $t_{r}$, is given by $\beta=\frac{f}{2} t_{r}^{3}$. Since $f_{0}$ is negative, $4 \mathrm{D}$ quantum geometry is asymptotically free. Here, note that, although background-metric independence includes an invariance under any confromal change of the background metric, the usual $\beta$-function is not needed to vanish. This nature is because there is a conformal anomaly, or the Wess-Zumino action in 4 dimensions [11, 10].

Consider the equation of motion for the conformal mode,

$$
-\frac{f}{(4 \pi)^{2}} \sqrt{-\bar{g}} \bar{C}^{2}-\frac{e}{(4 \pi)^{2}} \sqrt{-\bar{g}}\left(4 \bar{\Delta}_{4} \phi+\bar{G}-\frac{2}{3} \bar{\square} \bar{R}\right)+\frac{\delta}{\delta \phi} I_{E H}(g)=0 .
$$

Here, matter fields are conformally invariant so that there are no contributions to this equation of motion. The Einstein-Hilbert action has the following form:

$$
I_{E H}=6 m^{2} \int d^{4} x \sqrt{-\hat{g}} e^{\alpha \phi} \hat{\nabla}^{\lambda} \phi \hat{\nabla}_{\lambda} \phi+o\left(t_{r}^{2}\right),
$$

where, $\alpha$ is defined through the anomalous dimension of $m$ by the relation $\alpha=2+\gamma_{m}$. The lowest order of the anomalous dimension is computed by one-loop diagrams as $\gamma_{m}=\frac{\alpha^{2}}{4 e_{0}}[9]$. However, as for the minimal Standard Model with $N_{X}=4, N_{W}=45$ and $N_{A}=12$, for example, the anomalous dimension is sufficiently small as $\gamma_{m} \sim 1 / e_{0}=0.14$. Thus, in the following, we take the value of $\alpha$ approximately to be the classical one, $\alpha=2$.

Since the dynamics of the traceless mode is asymptotically free, in the high-energy region, the coupling, $t_{r}$, becomes small and we can neglect it. Further, we neglect the space-coordinate dependence of the conformal mode. We also consider the conformally flat metric, because it is simple and also the recent experiments suggest the flat universe [26], as

$$
d s^{2}=a(\eta)^{2}\left(-d \eta^{2}+\left(d x^{i}\right)^{2}\right) .
$$

Now, equation (8) has a quite simple form,

$$
-\frac{4 e}{(4 \pi)^{2}} \partial_{\eta}^{4} \log a+12 m^{2} a^{2}\left(\partial_{\eta}^{2} \log a+\left(\partial_{\eta} \log a\right)^{2}\right)=0
$$


Here, we introduce the proper time as $d \tau=a(\eta) d \eta$. and use the variable $H=\frac{\dot{a}}{a}$, where a dot denotes a derivative with respect to the proper time. We then obtain the equation of motion for $H$ as

$$
\dddot{H}+7 H \ddot{H}+4 \dot{H}^{2}+18 H^{2} \dot{H}+6 H^{4}-\frac{3 M^{2}}{e}\left(\dot{H}+2 H^{2}\right)=0,
$$

where $M=4 \pi m$. This equation has a de Sitter solution

$$
\dot{H}=0, \quad H=H_{0},
$$

where $H_{0}= \pm \frac{M}{\sqrt{e}}$. The plus sign gives an exponentially expanding solution, $a(\tau)=a_{0} e^{M \tau / \sqrt{e}}$.

Next, we discuss the stability of this solution. Consider a small deviation from the de Sitter solution as $H=H_{0}(1+\delta)$. Substituting this into equation (12) and neglecting $o\left(\delta^{2}\right)$ terms, we obtain

$$
\dddot{\delta}+7 H_{0} \ddot{\delta}+15 H_{0}^{2} \dot{\delta}+12 H_{0}^{3} \delta=0 .
$$

We here consider a solution of the type $\delta=e^{\kappa \tau}$. We then obtain the equation, $\kappa^{3}+7 H_{0} \kappa^{2}+15 H_{0}^{2} \kappa+12 H_{0}^{3}=0$. This equation has three solutions

$$
-4 H_{0}, \quad\left(-\frac{3}{2} \pm i \frac{\sqrt{3}}{2}\right) H_{0}
$$

For $H_{0}>0$, all solutions have $R e(\kappa)<0$. Thus, the de Sitter solution is stable.

Inflation must end at the low-energy region. In our model a classical limit is given by $e \rightarrow \infty$. The asymptotic freedom suggests that this limit will be realized at the low-energy region where the coupling, $t_{r}$, diverges. Large fluctuations of the gravitational fields freeze and small fluctuations ruled by the Einstein-Hilbert action survive at the low-energy region. Thus, the lifetime of the inflationary phase is given by the order of the inverse of the scale, $\mu$.

A stable inflationary scenario is also considered in [7], in which, however, there is no dynamical reason to end inflation because their argument is semiclassical.

There are some problems in our scenario. One is that we set the cosmological constant vanishing as in the original paper by Starobinsky, because 
whether the de Sitter solution exists or not depends on the initial value of the cosmological constant. The other is that we here restrict the solution of the equation of motion as in the form (10) where the space-coordinate dependence of the conformal mode is neglected. In our scenario, vanishing of the scalar curvature is realized by the dynamics of the traceless mode. This is different from a mechanism discussed in [9], in which the dynamics of the 4 derivative theory of the conformal mode gives a vanishing expectation value of the scalar curvature at the long distance. In any case, the dynamics of the gravitational fields would solve problems in inflationary cosmology.

\section{Acknowledgements}

This work is supported in part by the Grant-in-Aid for Scientific Research from the Ministry of Education, Science and Culture of Japan.

\section{References}

[1] A. Guth, Phys. Rev. D23 (1981) 347;

K. Sato, Mon. Not. R. Astr. Soc. 195 (1981) 467.

[2] A. Linde, Phys. Lett. B108 (1982) 389;

A. Albrecht and P. Steinhardt, Phys. Rev. Lett. 48 (1982) 1220.

[3] A. Starobinsky, Phys. Lett. 91B (1980) 99.

[4] A. Vilenkin, Phys. Rev. D32 (1985) 2511.

[5] S. Hawking, T. Hertog and H. Reall, Trace anomaly driven inflation, hep-th/0010232.

[6] S. Nojiri and S. Odintsov, AdS/CFT correspondence, conformal anomaly and quantum corrected entropy bounds, hep-th/0011115.

[7] J. Fabris, A. Pelinson and I. Shapiro, Grav. Cosmol. 6 (2000) 59 grqc/9810032; On the gravitational waves on the background of anomalyinduced inflation, hep-th/0009197; Anomaly-induced effective action and inflation, hep-th/0011030. 
[8] E. Tomboulis, Nucl. Phys. 329 (1990) 410.

[9] I. Antoniadis and E. Mottola, Phys. Rev. D45 (1992) 2013;

I. Antoniadis, P. Mazur and E. Mottola, Phys. Lett. B323 (1994) 284, B394 (1997) 49;

S. Odintsov, Z. Phys. C54 (1992) 531;

I. Antoniadis and S. Odintsov, Phys. Lett. B343 (1995) 76.

[10] K. Hamada, Integrability and Scheme-Independence of Even Dimensional Quantum Geometry Effective Action, hep-th/0012053.

[11] K. Hamada, Prog. Theor. Phys. 103 (2000) 1237 hep-th/9912098; hepth/0005063.

[12] J. Wess and B. Zumino, Phys. Lett. 37B (1971) 95.

[13] L. Bonora, P. Cotta-Ramusino and C. Reina, Phys. Lett. B126 (1983) 305 ;

L. Bonora, P. Pasti and M. Bregola, Class. Quant. Grav. 3 (1986) 635.

[14] D. Capper and M. Duff, Nuovo Cimento 23A (1974) 173.

[15] S. Deser, M. Duff and C. Isham, Nucl. Phys. B111 (1976) 45.

[16] M. Duff, Nucl. Phys. B125 (1977) 334;

Twenty years of the Weyl anomaly, Class. Quant. Grav. 11 (1994) 1387 hep-th/9308075 and references therein.

[17] R. Riegert, Phys. Lett. 134B (1984) 56.

[18] E. Fradkin and A. Tseytlin, Phys. Lett. 134B (1984) 187.

[19] A. Polyakov, Phys. Lett. 103B (1981) 207; Mod. Phys. Lett. A2 (1987) 893;

V. Knizhnik, A. Polyakov and A. Zamolodchikov, Mod. Phys. Lett. A3 (1988) 819;

J. Distler and H. Kawai, Nucl. Phys. B321 (1989) 509;

F. David, Mod. Phys. Lett. A3 (1988) 1651;

K. Hamada, Phys. Lett. B300 (1993) 322;

K. Hamada and A. Tsuchiya, Int. J. Mod. Phys. A8 (1993) 4897. 
[20] I. Antoniadis, P. Mazur and E. Mottola, Nucl. Phys. B388 (1992) 627.

[21] K. Hamada and F. Sugino, Nucl. Phys. B553 (1999) 283.

[22] H. Kawai, Y. Kitazawa and M. Ninomiya, Nucl. Phys. B393 (1993) 280.

[23] S. Deser and A. Schwimmer, Phys. Lett. B309 (1993) 279 hepth/9302047.

[24] S. Deser, Phys. Lett. B479 (2000) 315 hep-th/9911129.

[25] E. Fradkin and A. Tseytlin, Nucl. Phys. B201 (1982) 469; Phys. Lett. 104B (1981) 377.

[26] P. de Bernardis et al., Nature 404 (2000) 955 astro-ph/0004404. 